\title{
Proposed method for searches of gravitational waves from PKS 2155-304 and other blazar flares
}

\author{
S Desai ${ }^{1}$, K Hayama ${ }^{2}$, S D Mohanty ${ }^{2}$, M Rakhmanov ${ }^{2}$, \\ T Summerscales ${ }^{3}$, S Yoshida ${ }^{4}$ \\ 1 The Pennsylvania State University, University Park, PA 16802, USA \\ 2 The University of Texas, Brownsville, TX 78520, USA \\ 3 Andrews University, Berrien Springs, MI 49104, USA \\ 4 Southeastern Louisiana University, Hammond, LA 70402, USA \\ E-mail: desai@gravity.psu.edu
}

\begin{abstract}
.
We propose to search for gravitational waves from PKS 2155-304 as well as other blazars. PKS 2155-304 emitted a long duration energetic flare in July 2006 , with total isotropic equivalent energy released in $\mathrm{TeV}$ gamma rays of approximately $10^{45}$ ergs. Any possible gravitational wave signals associated with this outburst should be seen by gravitational wave detectors at the same time as the electromagnetic signal. During this flare, the two LIGO interferometers at Hanford and the GEO detector were in operation and collecting data. For this search we will use the data from multiple gravitational wave detectors. The method we use for this purpose is a coherent network analysis algorithm and is called RIDGE. To estimate the sensitivity of the search, we perform numerical simulations. The sensitivity to estimated gravitational wave energy at the source is about $2.5 \times 10^{55} \mathrm{ergs}$ for a detection probability of $20 \%$. For this search, an end-to-end analysis pipeline has been developed, which takes into account the motion of the source across the sky.
\end{abstract}

PACS numbers: 04.80.Nn, 95.85.Sz, 97.60.Lf, 98.54.Cm

\section{Introduction}

Blazars are one type of active galactic nuclei which are powered by accretion onto a central engine, and have been detected throughout the electromagnetic spectrum from radio waves to $\mathrm{TeV}$ gamma rays [1]. Blazars show strong variability on many different time scales throughout the electromagnetic spectrum and exhibit a high degree of polarization. Their radio jets exhibit apparent super-luminal motion indicating that they are emitted at small angles to our line of sight. The central engine is believed to consist of a super-massive black hole $\left(>10^{6} M_{\odot}\right)$ or a binary black hole [2]. There are more than 500 confirmed blazars and their mean redshift is about $0.2[3]$.

In many respects blazars are similar to gamma-ray bursts (GRBs), and there have been many searches for gravitational waves from GRBs [7. Both GRBs and blazars have a central engine and a jet, both undergo accretion, and both show evidence for non-thermal emission [4]. The main difference between them is the nature of the central engine, and that GRBs emit outbursts only once, whereas blazars emit outbursts more than once. 
Spatial correlations have been observed between blazars and ultra high energy cosmic rays [5. There have been searches for high energy neutrinos associated with blazar flares [6]. The benefits of coincidence searches between the data from neutrino detectors and gravitational wave detectors are outlined in 8]. This also motivates the search for gravitational waves in coincidence with blazar flares.

In this paper we present a method for searching for gravitational waves associated with an energetic outburst from PKS 2155-304 blazar, which happened during the fifth LIGO science run (S5) 10. Assuming that the velocity of gravitational waves is same as that of light, any possible gravitational wave signals should be seen simultaneously along with the electromagnetic outburst. Therefore, this search needs to be done by analyzing the data from gravitational wave detectors at the time of this flare.

To do this search, we shall combine the data from many gravitational wave detectors. The search will be done with a coherent network analysis algorithm called RIDGE [9]. Sensitivity results using simulated noise and one set of simulated signals are presented. We also briefly discuss the outbursts seen from OJ 287 and S5 0716+71, which are two other blazars we plan to search for gravitational waves.

Since the flare from PKS 2155-304 lasted several hours, the search for gravitational wave signals from this object has features that are quite distinct from previous LIGO searches for gravitational waves associated with GRBs. For this search, we need to analyze a much longer data segment, during which the source moves significantly on the sky. This is different from searches for gravitational waves from GRBs, where the on-source time interval for which data is analyzed for possible gravitational wave signals is only 180 seconds [7]. For this search, the corresponding change in the detector responses due to the changing antenna pattern functions must be taken into account.

\section{PKS 2155-304 outburst in July 2006}

PKS 2155-304 is a blazar located in the southern galactic hemisphere with redshift of about 0.12 , which corresponds to a luminosity distance of about $540 \mathrm{Mpc}$ [11, and has been observed throughout the electromagnetic spectrum. The nature of the central engine in PKS 2155-304 is currently unknown. The High Energy Stereoscopic System (H.E.S.S) [12 detector has been monitoring PKS 2155-304 since 2002. It detected an energetic outburst from PKS 2155-304 [13] above $200 \mathrm{GeV}$, which lasted about 2000 seconds, starting from MJD = 53944 (corresponding to July 282006 00:40:00 UTC). The total flux during this flare increased by a factor of ten with respect to the quiescent level. The integrated flux during this flare is $1.7 \times 10^{-9} \mathrm{~cm}^{-2} \mathrm{sec}^{-1}$. The total isotropic equivalent energy emitted during this flare assuming the source has a redshift of about 0.12 , matter density of 0.3 , density of cosmological constant of 0.7 , is approximately $10^{45}$ ergs.

Various models have been proposed for this outburst [14, 15, 16, but none of them could satisfactorily explain this large flare along with the steady state emission from this object. The common feature of all the models used to explain the flare is that the bulk Lorentz factor of the jet is around 50, size of the emitting region is around $3 \times 10^{14} \mathrm{~cm}$, and the jet opening angle is about $1^{\circ}$, which reduces the isotropic equivalent energy emitted by a factor of about $10^{-4}[17$.

During this outburst from PKS 2155-304, both the $4 \mathrm{~km}$ (H1) and $2 \mathrm{~km}$ (H2) LIGO Hanford interferometers and the GEO detector were operational. The third LIGO detector in Livingston, LA was not taking data at this time. The angular 


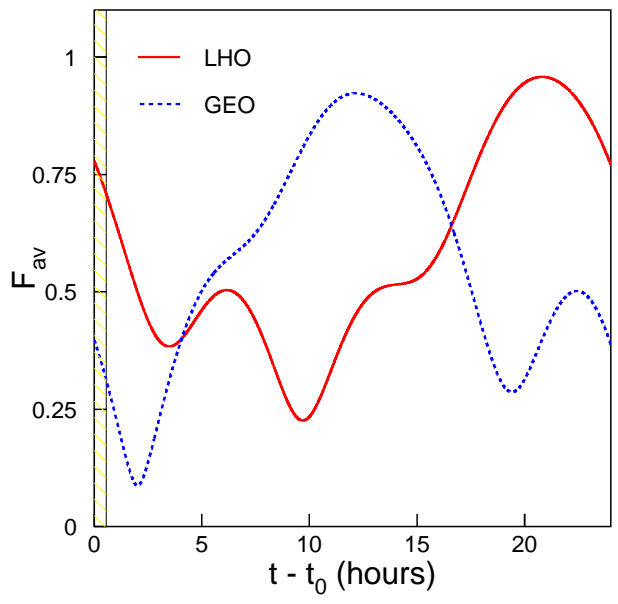

Figure 1. The detector response function $\left(F_{a v}=\sqrt{F_{+}^{2}+F_{\times}^{2}}\right)$ of the LIGO Hanford detectors and GEO detector in a 24 hour interval starting from the PKS 2155-304 flare, which corresponds to GPS time $\left(t_{0}\right)$ equal to 838082414. The hatched region shows the duration of the flare

sensitivities to unpolarized gravitational waves of $\mathrm{H} 1, \mathrm{H} 2$, and $\mathrm{GEO}$ within a one day interval from the start of this flare, are shown in figure 1. The sensitivity of gravitational wave interferometers can be characterized by its Inspiral Range [18], defined as the distance to which signals from the inspiral of two $1.4 M_{\odot}$ neutron stars is detected with signal-to-noise ratio greater than 8 , after averaging over all sky positions and orientations of the binary system. The corresponding Inspiral Range for H1, H2, and GEO detectors were $13 \mathrm{Mpc}, 7 \mathrm{Mpc}$ and $1.3 \mathrm{Mpc}$ respectively during this time. This is at least 50 times smaller than the distance to PKS 2155-304.

\section{Mechanisms for possible gravitational wave emission}

There are no theoretically accepted gravitational wave emission mechanisms from such transient blazar outbursts in the LIGO frequency band. There have been some proposed mechanisms for steady state gravitational wave emission from Active Galactic Nuclei with super-massive black holes as the central engine in the LISA frequency band, which we briefly describe here. We should point out that the distance to this object is much greater than the Inspiral Range of the LIGO and GEO detectors (Section 2) at this time, and a large amount of gravitational wave energy must be emitted if the expected signal is near the sensitivity of initial LIGO. Ultimately, since the cause of this flare is unknown, we would like to be open to all possibilities and look for gravitational waves during this outburst without any theoretical prejudice.

One mechanism is through the fragmentation of the accretion disk due to feedback energy from star formation in the outer parts of the accretion disk for a blazar with single black hole [19] or due to impact from the accretion disk of the secondary black hole for a blazar with binary black hole [20. Another mechanism is through the fragmentation of the accretion disk because of dynamical friction from an infalling satellite onto a coplanar accretion disk 21]. This fragmentation results in 
the formation and evolution of massive stars in the self-gravitating accretion disks of massive black holes. The resultant compact objects from the fragmentation remain embedded in the accretion disk and could merge with the parent black hole at the center 19. The gravitational waves are possibly produced from inspirals and mergers of the disk born compact objects (with masses of about $100 M_{\odot}$ ) with the central black hole. The expected strain amplitude for the above mechanisms has been estimated to be from $10^{-21}$ to $10^{-19} \mathrm{~Hz}^{-1 / 2}$ with expected frequency from $10^{-4}$ to $10^{-1} \mathrm{~Hz}[22$. If a less massive object is formed, the expected frequency of the gravitational wave signal would be higher and could fall into the LIGO band. However, a less massive object will also lead to a significant reduction in the amount of gravitational wave emission.

\section{Analysis techniques}

Since the LIGO Hanford and GEO detectors are far apart, we will use gravitational wave coherent network analysis techniques for analyzing this flare. Such coherent network analysis algorithms take into account the antenna patterns for each detector in order to extract the gravitational wave signal from the detector data stream 23 , 24, 25, 26. The RIDGE [9] algorithm (the name is derived from the term "ridge regression" used in statistics literature) is one such method. The technique solves the problem of rank deficiency of the antenna response matrix by using Tikhonov regularization [23. More details on RIDGE and the data conditioning technique used can be found in [27. We also propose to use the same method in searches for gravitational waves from GRBs, magnetars, pulsar glitches, and Sco-X1. For this analysis we would like to combine the data from the GEO detector with the data from the two Hanford detectors. The GEO detector is geographically far apart and has different arm orientations with respect to the Hanford detectors. Therefore, using GEO data would enable us to increase the sensitivity in contrast to searches for gravitational waves from GRB 070201 [28] or SGR 1806-20 29], which used the data from only the Hanford detectors.

We are also developing a method to look for long-duration (lasting several seconds to minutes) transient gravitational wave signals [31, taking into account the motion of the source in the sky. This method of source tracking involves splitting the dataset into many short duration segments during which the position of the source is approximately constant. We reconstruct $h_{+}$and $h_{\times}$within each segment knowing the direction to the source. The reconstructed $h_{+}$and $h_{\times}$is then concatenated across the segments and a test for excess power is applied to this reconstructed time-series. Since the flare from PKS 2155-304 lasted for more than two hours, we plan to apply this source tracking technique. This method of looking for long duration unmodelled bursts in LIGO data could help us find a signal which otherwise may be missed from all sky untriggered searches for gravitational wave bursts [30], which will also analyze the data during this flare.

\section{Estimation of sensitivity of the algorithm}

In order to evaluate the performance of this algorithm, we perform numerical simulations of the detector noise and possible gravitational wave signals. We then apply the RIDGE algorithm on this simulated time-series which includes both the 
detector noise and signal. We shall then estimate the sensitivity of our search. using Receiver Operating Characteristic curves.

Since the flare from PKS 2155-304 lasted 2000 seconds, we need to generate the same duration of simulated noise for H1, H2, and GEO. The amplitude spectral density of the simulated noise time series is obtained from the LIGO and GEO design curves.

We first generate 2000 seconds of Gaussian stationary noise using three independent realizations of white noise corresponding to the three detectors. We then created FIR filters with transfer functions matching the design amplitude noise spectral density curves as a function of frequency, for the three instruments. These were applied to the generated stationary Gaussian noise. To generate a realistic simulation of the detector noise we also need to add possible instrumental lines. To model the effect of such lines, sinusoidal signals were added at seven frequencies between 50 and $1050 \mathrm{~Hz}$. The amplitude spectral density of the simulated noise for LHO and GEO is shown in figure 2,

In order to test the performance of this method to possible short-duration gravitational wave signals from PKS 2155-304, we need to add simulated burst signals to the generated noise at various times throughout the flare. In its simplest form, a burst signal can be described by a sine-Gaussian waveform, with quality factor $Q$, central frequency $\left(f_{0}\right)$ and a characteristic amplitude [32. The waveforms we chose for our simulation were circularly polarized sine-Gaussian signals with $Q=9$ and $f_{0}=235 \mathrm{~Hz}$, and for which the signal in ' + ' polarization is phase shifted by $90^{\circ}$ with respect to the signal in ' $x$ ' polarization. The strength of the signal is characterized by

$$
h_{\mathrm{rss}}^{2}=\int_{-\infty}^{\infty} \mathrm{d} t\left(h_{+}^{2}(t)+h_{\times}^{2}(t)\right),
$$

where $h_{+}$and $h_{\times}$correspond to waveforms with the ' + ' and ' $\times$' polarizations respectively. We chose four different values for the signal strength with $h_{\text {rss }}$ equal to $(0.7,1.4,2.1,3.5) \times 10^{-22} \mathrm{~Hz}^{-1 / 2}$, This signal was injected at the estimated location of PKS 2155-304, which is Right Ascension $=22$ hour and Declination $=-29.8^{\circ}$ [11.

The RIDGE method is now applied to our simulated dataset. At each value of latitude $(\theta)$ and longitude $(\phi)$, we maximize the Tikhonov regularized likelihood functional [23, by varying $h_{\times}$and $h_{+}$. To distinguish between signals and noise, we define a detection statistic using the values of the likelihood functional (S), and is called radial distance statistic 9 ]

$$
R_{\mathrm{rad}}=\left[\left(\frac{\max _{\theta, \phi} \mathbf{S}}{\max _{\theta, \phi} \overline{\mathbf{S}_{\mathbf{0}}}}-1\right)^{2}+\left(\frac{\max _{\theta, \phi} \mathbf{S}}{\min _{\theta, \phi} \mathbf{S}} \times \frac{\max _{\theta, \phi} \overline{\mathbf{S}_{\mathbf{0}}}}{\min _{\theta, \phi} \overline{\mathbf{S}_{\mathbf{0}}}}-1\right)^{2}\right]^{1 / 2}
$$

where $\overline{\mathbf{S}_{\mathbf{0}}}$ is the average likelihood of all skymaps that do not contain a signal.

In figure 3, we show plots of two skymaps in which the colour scale indicates the values of the likelihood functional. The first plot is the skymap at the maximum of the radial distance statistic which contains a signal. The second plot is the skymap averaged over all times not containing any signal. The pattern in the skymap is largely determined by the condition number of the LHO-GEO response matrix [23. The values of the radial distance statistic for the signal only and noise-only skymaps are shown in figure 4 .

A common way to characterize the sensitivity of an algorithm is through Receiver Operating Characteristic (ROC) curves of detection efficiency versus false alarm probability. The ROC curves of this search with the H1-H2-GEO network for three 


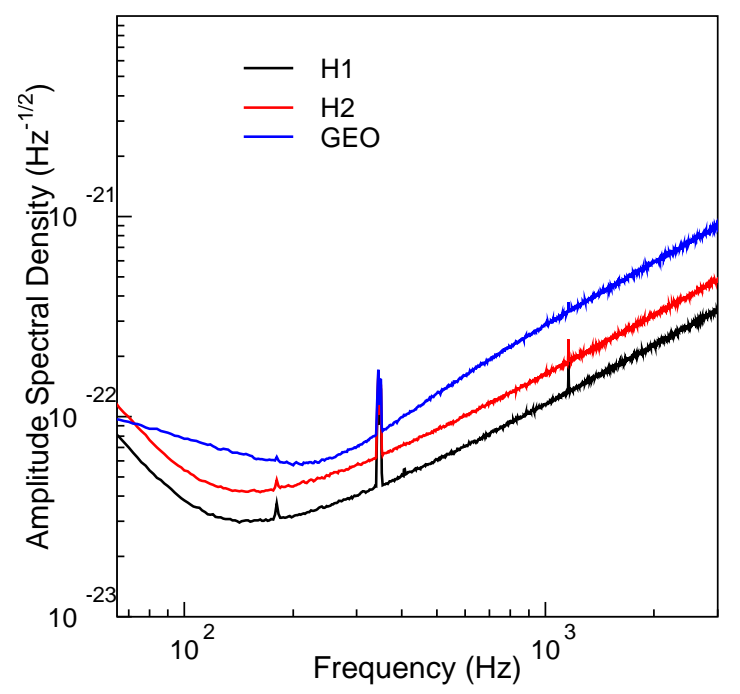

Figure 2. The amplitude spectral density of simulated data for the $4 \mathrm{~km}$ and $2 \mathrm{~km}$ Hanford interferometers (H1 and $\mathrm{H} 2$ ) and GEO detector (assuming signal detuning frequency of $200 \mathrm{~Hz}$ ) as a function of frequency.

values of $h_{\mathrm{rss}}$ are shown in figure 5. These ROC curves are obtained by calculating the detection probability and false alarm probability for different threshold values of the radial distance statistic. For a detection probability of 0.2 and false alarm probability of 0.004 , the $h_{\text {rss }}$ is about $2.1 \times 10^{-22} \mathrm{~Hz}^{-1 / 2}$. The energy for a sine-Gaussian signal with central frequency $\left(f_{0}\right)$ and $Q \gg 1$ is given by 32 ]

$$
E_{G W}=\frac{r^{2} c^{3}}{4 G}\left(2 \pi f_{0}\right)^{2} h_{\mathrm{rss}}^{2},
$$

where $r$ is the distance to the source. From (3), the $h_{\mathrm{rss}}$ sensitivity corresponds to total energy in gravitational waves at the source of approximately $2.5 \times 10^{55}$ ergs. Thus, the total energy emitted in gravitational waves from this object must be about $10^{10}$ greater than the emitted electromagnetic energy, in order to be sensitive to our method.

\section{Outbursts from OJ 287 and S5 $0716+71$}

In addition to PKS 2155-304, there were other blazar outbursts during S5, two of which we will describe here. We shall also look for possible gravitational waves from these two blazars.

OJ 287 is a blazar located at a redshift of about 0.3 , equivalent to luminosity distance of about $1600 \mathrm{Mpc}$. OJ 287 has been known to emit periodic outbursts every 12 years. This blazar is believed to contain a binary black hole system with masses equal to $16 \times 10^{9} M_{\odot}$ and $0.1 \times 10^{9} M_{\odot}[20$. The possible cause of these bursts is due to the fragmentation of the accretion disk of the secondary black hole by the primary one. The orbital decay of the system due to the emission of gravitational radiation (with frequency approximately equal to $10^{-8} \mathrm{~Hz}$ ) is in agreement with general relativity to 

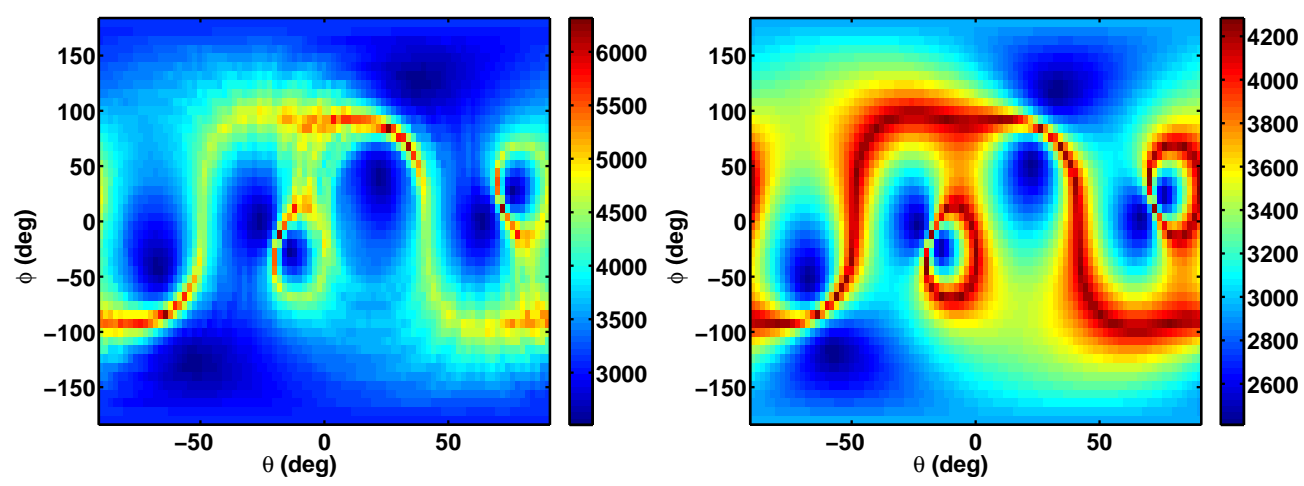

Figure 3. Likelihood skymap for a signal with $h_{\mathrm{rss}}=3.5 \times 10^{-22} \mathrm{~Hz}^{-1 / 2}$ (left) and for noise only values (right) as a function of latitude $(\theta)$ and longitude $(\phi)$.

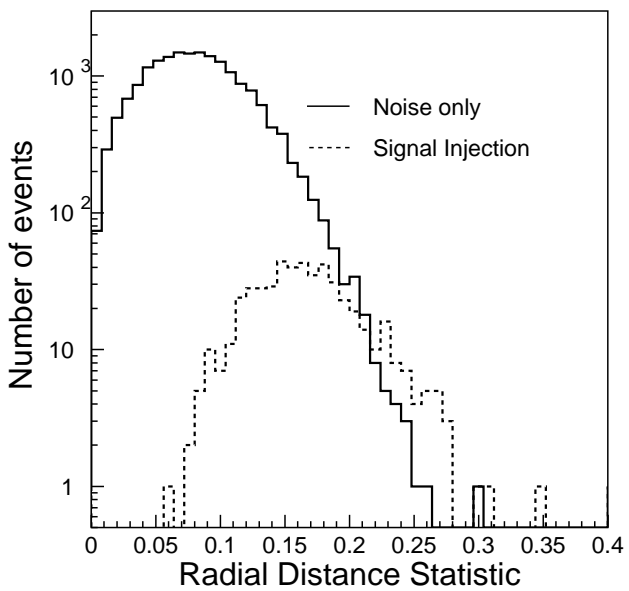

Figure 4. The distributions of the radial distance statistic (2) for both simulated signals (dashed line) and noise (solid line). The strength of the injected signal is $h_{\mathrm{rss}}=2.1 \times 10^{-22} \mathrm{~Hz}^{-1 / 2}$. The mean value of the radial distance statistic for signal injections is higher than for pure noise.

within $10 \%$ accuracy 33 . There were two optical outbursts from OJ 287 during S5, in November 2005 and September 2007. Figure 6 shows one of the bursts from OJ 287 in November 2005 in the optical $V$ band.

Another blazar for which outbursts were observed in the radio, optical and gamma-ray bands is S5 $0716+71$. Not much is known about the redshift of this system or the nature of its central engine. These outbursts were detected from AugustSeptember 2007 by the Whole Earth Blazar Telescope Consortium using data from the AGILE satellite in MeV gamma rays, optical observations in $R$-band, and radio observations from 8 to $43 \mathrm{GHz}$ [34. The cause of these outbursts is unknown.

We shall also apply RIDGE to search for possible short-duration as well as longduration gravitational wave signals from OJ 287 and S5 $0716+71$. For these objects, since there were few observations per day, the on-source time interval is expected to 


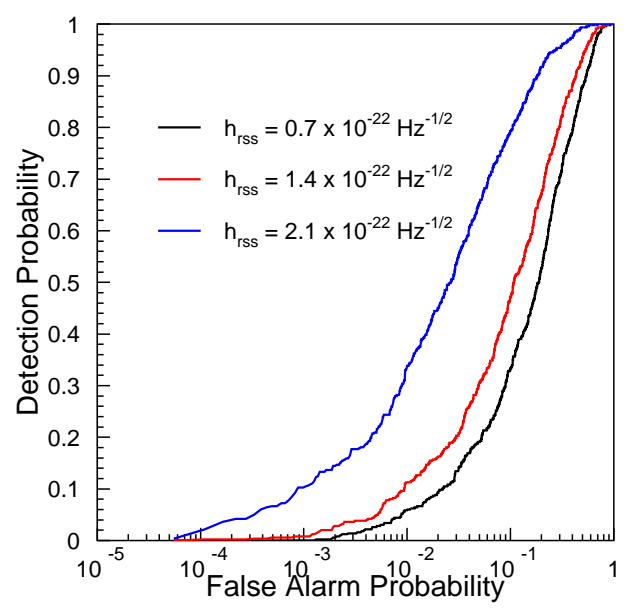

Figure 5. Receiver Operating Characteristic (ROC) curve for H1-H2-GEO network with the RIDGE pipeline during the PKS 2155-304 flare for a circularly polarized sine-Gaussian signal for three different values of $h_{\mathrm{rss}}$ shown in the figure in units of $\mathrm{Hz}^{-1 / 2}$.

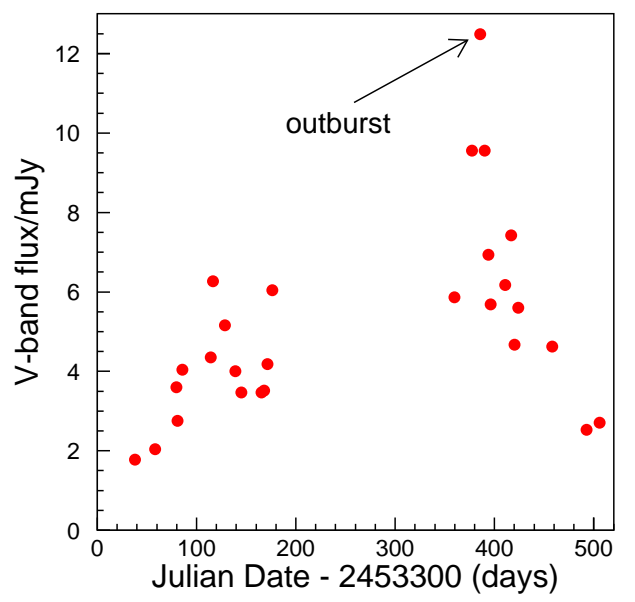

Figure 6. Observations from OJ 287 starting from 21 October 2004 12:00 UTC. The outburst is in November 2005 and corresponds to $V$-band flux of about 12 mJy. (Data used for this plot is obtained from [20].) 
be several days.

\section{Conclusions}

We described a method to search for gravitational waves from blazars and identified some sources which flared in $\mathrm{TeV}$ gamma rays and optical wavelengths, such as PKS 2155-304, OJ 287, and S5 0716+71. We discussed how a coherent network analysis algorithm (RIDGE) can be used for this search. Sensitivity results with the RIDGE search algorithm, using simulated noise and simulated signals for the LIGO Hanford detectors and GEO detector, at the time of the PKS 2155-304 flare are shown. This algorithm can detect $h_{\mathrm{rss}}$ of about $2.1 \times 10^{-22} \mathrm{~Hz}^{-1 / 2}$ for a detection probability of about $20 \%$. This corresponds to an energy estimate at the source of $2.5 \times 10^{55}$ ergs for the assumed signal waveform and should be of the same order of magnitude for any other short-duration burst signal. A complete end-to-end pipeline to do this search for gravitational waves from these blazar flares as well as other long-duration electromagnetic transients, which takes into account the motion of the source across the sky has been designed.

\section{Acknowledgments}

We would like to thank M Eracleous, R Frey, M Hewitson, P Kalmus, S Marka, N Leroy, L Cadonati, P Sutton, W Cui, G Ghishellini, K Nilsson, and M Valtonen for many fruitful discussions and valuable comments on this paper. We are also indebted to W Steffen for providing permission to show his animation in our poster at the GWDAW 12 workshop. This work is supported by NSF 428-51 29NV0 and Center for Gravitational Wave Physics (PSU), NSF PHY-055584 and NASA NAG5-13396 (UTB), NSF PHY-0653233 (SELU), Office of Scholarly Research (Andrews). The Center for Gravitational Wave Physics is funded by the National Science Foundation under Cooperative Agreement PHY 01-14375. This paper was assigned LIGO document number P080023.

\section{References}

[1] Urry C M and Padovani P 1995 Publ. Astron. Soc. Pac. 107803

[2] Hayasaki K, Mineshige K and Sudou S 2007 Publ. Astron. Soc. Japan 59427

[3] Turriziani S, Cavazzuti E and Giommi P 2007 Astron. Astrophys. 472, 699

[4] Ghisellini G 2006 Preprint astro-ph/0611077

[5] Gorbunov D S et al 2006 J. Cosmol. Astropart. Phys. 060125

[6] Abe K et al (The Super-Kamiokande Collaboration) 2006 Astrophys J. 652198

[7] Abbott B et al (The LIGO Scientific Collaboration) 2007 Preprint arXiv:0709.0766

[8] Aso Y et al 2008 Class. Quantum Grav. 25114039

[9] Hayama K, Mohanty S D, Rakhmanov M and Desai S 2007 Class. Quantum Grav. 24 S681

[10] Abbott B et al (The LIGO Scientific Collaboration) 2007 Preprint arXiv:0711.3041

[11] Chadwick P M et al 1999 Astrophys. J. 513161

[12] Aharonian F et al 2004 Nature 43275

[13] Aharonian F et al 2007 Astrophys. J. 664 L71

[14] Begelman M C Fabian A C and Rees M J 2007 Mon. Not. R. Astron. Soc. 384 L19

[15] Ghisellini G and Tavecchio F 2008 Mon. Not. R. Astron. Soc. 386 L28

[16] Finke J D, Dermer C D and Bottcher M 2008 Preprint arXiv:0802.1529

[17] Kulkarni S R et al 1999 Nature 398389

[18] Sutton P J 2003 LIGO Technical Report T030276

[19] Levin Y 2003 Preprint astro-ph/0307084

[20] Valtonen M J et al 2006 Astrophys. J. 643 L9 
[21] Chang P 2008 Preprint arXiv:0801.2133

[22] Sigl G, Schnittman J and Buonnano A 2007 Phys. Rev. D 75024034

[23] Rakhmanov M 2006 Class. Quantum Grav. 23 S673

[24] Chatterji S et al 2006 Phys. Rev. D 74082005

[25] Klimenko S, Yakushin I, Mercer A and Mitselmakher G 2008 Class. Quantum Grav. 25114029

[26] Kalmus P, Khan R, Matone L and Màrka S 2007 Class. Quantum Grav. 24 S659

[27] Hayama K et al 2008 Class. Quantum Grav. (in this volume)

[28] Abbott B et al (The LIGO Scientific Collaboration) 2008 Astrophys. J. (in press) Preprint arXiv:0711.1163

[29] Abbott B et al (The LIGO Scientific Collaboration) 2007 Phys. Rev. D 76062003

[30] Abbott B et al (The LIGO Scientific Collaboration) 2007 Class. Quantum Grav. 245343

[31] Hayama $\mathrm{K}$ et al 2008 TAUP proceedings (in press)

[32] Abbott B et al (The LIGO Scientific Collaboration) 2007 Class. Quantum Grav. 245343

[33] Valtonen M J et al 2008 Nature 452851

[34] Villata M et al 2008 Astron. Astrophys. 481, L79 